\title{
ANÁLISE DO CUSTO E DO RAIO ECONÔMICO DE TRANSPORTE DE MADEIRA DE REFLORESTAMENTOS PARA DIFERENTES TIPOS DE VEÍCULOS ${ }^{1}$
}

Márcio Lopes da Silva², Robson José de Oliveira ${ }^{3}$, Sebastião Renato Valverde², Carlos Cardoso Machado²

e Vanessa Aparecida Vieira Pires ${ }^{3}$

\begin{abstract}
RESUMO - Este estudo foi realizado com o objetivo de estabelecer a distância máxima de transporte viável para cada tipo de veículo utilizado para transportar a madeira das áreas de colheita até centros de consumo. Para tanto, utilizaram-se dados de custos e receitas de um reflorestamento, bem como a capacidade de carga de diferentes composições veiculares empregadas no transporte. Aplicando-se os critérios econômicos (VPL, TIR, CMP e BCPE), as distâncias variaram entre 155 e 226 km, para o caminhão-truck e o rodotrem, respectivamente. Concluiu-se que o rodotrem pode alcançar distância maior de transporte, sendo o preço da madeira a variável que mais influenciou a distância máxima de transporte.
\end{abstract}

Palavras-chave: Distância, transporte e economia florestal.

\section{COST AND DISTANCE OF REFORESTATION WOOD TRANSPORT FOR DIFFERENT TYPES OF TRUCKS}

\begin{abstract}
The objective of this study was to establish the viable transport maximum distance for each type of wood transportation vehicle from the harvest areas to the consumption centers. Thus, reforestation cost and revenue data were used, as well as the load capacity of different types of vehicles used for wood transport. The application of economic criteria (VPL, TIR, CMP and BCPE) showed that the distances varied between 155 and $226 \mathrm{~km}$, for the small truck and rodotrem (truck with two large trailers), respectively. It was concluded that rodotrem could reach a greater distance, with wood price being the factor most influencing maximum transport distance.
\end{abstract}

Keywords: Distance, transport and forest economy.

\section{INTRODUÇÃO}

O setor florestal tem sido um dos mais importantes da economia nacional. Em 2003, contribuiu com 4\% do PIB, $8 \%$ das exportações brasileiras, recolhimento de US\$ 2 bilhões de impostos e 2 milhões de empregos (SBS, 2004). Além disso, o setor florestal apresenta grande potencial de crescimento. Prova disso são os investimentos das empresas de base florestal, tanto na área industrial, ampliando suas capacidades instaladas, quanto na compra de novas áreas para plantios de florestas e aquisição de máquinas e equipamentos mais eficientes, buscando sempre a otimização do processo produtivo, desde o plantio e manutenção até a colheita e transporte florestal. Tudo isso, com o intuito de redução de custos, pela economia de escala (NOCE et al., 2005).

O transporte florestal consiste na movimentação de madeira dos pátios ou das margens das estradas

\footnotetext{
${ }^{1}$ Recebido em 20.12.2006 e aceito para publicação em 04.07.2007.

${ }^{2}$ Departamento de Engenharia Florestal da Universidade Federal de Viçosa (UFV). E-mail: <marlosil@ ufv.br>.

${ }^{3}$ Programa de Pós-Graduação em Ciência Florestal da UFV. E-mail: <robinhojo@ yahoo.com.br>.
} 
nos talhões até o local de consumo ou pátio das empresas. No Brasil, pode ser realizado por diversos meios (ferroviário, dutoviário, rodoviário), em que este último representa $85 \%$ de toda a madeira que é transportada e, ainda, $62 \%$ de todos os produtos transportados no país. Mesmo com as condições precárias de trafegabilidade em que se encontram muitas estradas, além de serem essas o único meio capaz de interligar as indústrias às suas fontes de abastecimento de madeira, localizadas em origens distintas, ou seja, abrangendo áreas descontinuas dentro do Brasil (STEIN et al., 2001). Por isso, estudos buscando maior eficiência no transporte rodoviário florestal são muito importantes.

A madeira é um insumo (ou produto) de baixo valor específico, ou seja, o seu valor em relação ao seu peso e, ou, volume é baixo. Por isso, o custo de transporte dessa mercadoria é relativamente alto. $O$ custo de transporte varia diretamente com a distância percorrida. Por isso, a localização da fábrica e dos reflorestamentos são decisões estratégicas e requerem planejamento detalhado.

Quanto mais longo o trajeto percorrido, maior será o custo unitário por volume de madeira transportada (SILVERSIDES, 1976). Uma das maneiras de diminuir o custo em percursos longos tem sido a utilização de veículos com maior capacidade de carga (LEITE, 1992).

Atualmente, o número de eixos na composição e o peso em cada eixo são fatores de preocupação para o transportador que busca o otimização da quantidade de madeira transportada por veículo sem, contudo, ultrapassar os limites estabelecidos pela legislação (SEIXAS, 2001).

Vários fatores influenciam o transporte de cargas pelo modal rodoviário, e, no caso florestal, isso não poderia ser diferente. Ganham destaque os tipos de veículo, a distância de transporte, o valor unitário do frete, as condições em que se encontram a malha rodoviária, o tempo de espera no carregamento e descarregamento, a capacidade de carga em volume que o veículo transporta, as condições locais e regionais e os tipos de equipamentos de carregamento e descarregamento (MACHADO et al., 2000).

Os veículos utilizados no transporte florestal rodoviário podem ser classificados de acordo com a capacidade de carga. Assim, têm-se os veículos leves (que têm capacidade de carga que não ultrapassa 10 toneladas); veículos pesados (que suportam de 30 a 40 toneladas de carga); e veículos extrapesados (com capacidade de carga acima das 40 toneladas). Como exemplo de veículos extrapesados, tem-se o bitrem (um cavalo mecânico e dois semi-reboques), tritrem (um cavalo mecânico e três semi-reboques), treminhão (um caminhão e dois reboques) e o rodotrem (um veículo articulado e um reboque) (MACHADO et al., 2000). Porém, estudos devem ser conduzidos para identificar qual o tipo de veículo mais indicado para se transportar madeira de determinada região ou empresa.

Outra grande dúvida das empresas é com relação à distância máxima viável de transporte de madeira. Pois o custo de transporte elevado pode inviabilizar reflorestamentos localizados mais distantes dos centros de consumo.

E, por último, as variáveis econômicas como preço da madeira, produção florestal, custos relacionados ao projeto florestal e taxa de desconto, dentre outras, afetam a lucratividade do reflorestamento e, conseqüentemente, a distância máxima viável de transporte.

Com base nessas questões, este trabalho objetivou estabelecer a distância máxima de transporte de madeira e de um reflorestamento até um centro de consumo, para cada tipo de veículo, bem como identificar quais variáveis econômicas mais afetam essa distância.

\section{MATERIAL E METODOS}

\subsection{Dados do projeto de reflorestamento}

Para alcançar os objetivos propostos neste trabalho, considerou-se um produtor independente que pretende implantar um reflorestamento e entregar a madeira na fábrica. Ele quer saber qual a distância máxima de transporte ou o raio econômico de transporte. Para isso, utilizaram-se dados reais de um projeto de reflorestamento (Quadro 1).

Quadro 1 - Dados de um empreendimento florestal Table 1 - Data of a forest project

\begin{tabular}{lc}
\hline \multicolumn{1}{c}{ Itens } & Valores \\
\hline Custo de implantação & $800.00 \mathrm{US} \$ / \mathrm{ha}$ \\
Custo de manutenção ano 1 & $120.00 \mathrm{US} \$ / \mathrm{ha}$ \\
Custo de manutenção ano 2 & $70.00 \mathrm{US} \$ / \mathrm{ha}$ \\
Custo de roçada pré-corte no ano 7 & $45.00 \mathrm{US} \$ / \mathrm{ha}$ \\
Custo de manutenção anual & $60.00 \mathrm{US} \$ / \mathrm{ha}$ \\
Custo de colheita no ano 7 & $3.50 \mathrm{US} \$ / \mathrm{m}^{3}$ \\
Produção aos 7 anos & $280 \mathrm{~m}^{3} / \mathrm{ha}$ \\
Preço da terra & $750.00 \mathrm{US} \$ / \mathrm{ha}$ \\
Preço da madeira & $24 \mathrm{US} \$ / \mathrm{m}^{3}$ \\
Taxa de juros & $8 \% \mathrm{a} . \mathrm{a}$. \\
\hline
\end{tabular}




\subsection{Classificação dos tipos de veículos}

No Quadro 2, mostram-se os modelos de vários tipos de veículos que são utilizados no transporte florestal, considerados neste estudo com as respectivas capacidades de carga.

\subsection{Calculo do custo de transporte pelos diferentes tipos de veículos}

Para o calculo do custo de transporte de cada um dos veículos utilizados (Quadro 2), empregou-se a seguinte fórmula:

$$
C T=\frac{P f}{C a p} * 2 D * P
$$

em que:

$$
\mathrm{CT}=\text { custo de transporte (US\$); }
$$

$\mathrm{Pf}=$ fator indicativo do preço médio do frete no mercado por $\mathrm{km}$ (US\$);

Cap = capacidade de carga $\left(\mathrm{m}^{3}\right)$;

2 = distância multiplicada por 2 , pois considera a viagem de ida e volta;

$$
\begin{aligned}
& \mathrm{D}=\operatorname{distância~}(\mathrm{km}) ; \mathrm{e} \\
& \mathrm{P}=\operatorname{produção~}\left(\mathrm{m}^{3} / \mathrm{ha}\right) .
\end{aligned}
$$

Esta fórmula (1) é uma alternativa a ser utilizada quando não se tem o custo hora do veículo. Optouse pelo seu uso, pois foi considerado que o produtor terceiriza o transporte e paga pelo frete, cujo valor médio do frete no mercado é de US\$1,5/km.

\subsection{Análise econômica}

Com os dados do projeto florestal e dados do custo do transporte pelos diferentes veículos, foi possível proceder a uma análise econômica e verificar a distância que cada veículo consegue transportar a madeira sem que haja prejuízo. A análise econômica foi realizada com base nos seguintes critérios de avaliação de projetos:

$$
\begin{aligned}
& \text { - Valor Presente Líquido (VPL); } \\
& \text { - Custo Médio de Produção (CMP); e } \\
& \text { - Taxa Interna de Retorno (TIR). }
\end{aligned}
$$

\subsubsection{Valor presente líquido (VPL)}

É a diferença entre o valor presente das receitas e o valor presente dos custos a determinada taxa de desconto.

$$
V P L=\sum_{j=0}^{n} R_{j}(1+i)^{-j}-\sum_{j=0}^{n} C_{j}(1+i)^{-j}
$$

em que:

$$
\begin{aligned}
& \mathrm{R}_{\mathrm{j}}=\text { receita ocorrida no período } \mathrm{j}(\mathrm{US} \$ / \mathrm{ha}) ; \\
& \mathrm{C}_{\mathrm{j}}=\text { custo ocorrido no período } \mathrm{j}(\mathrm{US} \$ / \mathrm{ha}) ; \\
& \mathrm{i}=\text { taxa de juros (\% ao ano); } \\
& \mathrm{j}=\text { período de ocorrência de receita ou custo (anos);e } \\
& \mathrm{n}=\text { número máximo de períodos (anos). }
\end{aligned}
$$

\subsubsection{Custo médio de produção (CMP)}

Consiste em dividir o valor atual do custo pela produção total equivalente (US $\$ / \mathrm{m}^{3}$ ):

$$
C M P=\frac{\sum_{j=0}^{n} C_{j}(1+i)^{-j}}{\sum_{j=0}^{n} P T_{j}(1+i)^{-j}}
$$

em que:

$$
\begin{aligned}
& \mathrm{CT}_{\mathbf{j}}=\text { custo ocorrido no período } \mathrm{j} ; \mathrm{e} \\
& \mathrm{PT}_{\mathbf{j}}=\text { produção total ocorrida no período j. }
\end{aligned}
$$

Produção equivalente é o somatório das quantidades produzidas em cada período $\mathrm{j}$, descapitalizadas para o período zero, considerando-se a taxa de juros.

\subsubsection{Taxa Interna de Retorno (TIR)}

É a taxa de juros que iguala o valor presente das receitas ao valor presente dos custos, ou seja, é a taxa que iguala o VPL a zero. Também, pode ser entendido como a taxa percentual do retorno do capital investido. Portanto, sua fórmula é dada por:

$$
\sum_{j=0}^{n} R_{j}(1+T I R)^{-j}=\sum_{j=0}^{n} C_{j}(1+T I R)^{-j}
$$

em que:

TIR = Taxa Interna de Retorno (ao ano).

As demais variáveis já foram definidas.

\subsection{Cálculo da distância máxima de transporte}

Aumentando-se a distância (D) da fórmula 1, o custo de transporte se eleva até chegar a um momento que inviabiliza o projeto (quando o VPL se torna negativo).

R. Árvore, Viçosa-MG, v.31, n.6, p.1073-1079, 2007 
Portanto a distância máxima de transporte é aquela que anula o VPL, também conhecida como ponto de nivelamento da distância (SILVA et al., 2005). Este procedimento foi realizado utilizando a ferramenta "atingir metas" da planilha eletrônica (Excel).

\subsection{Análise de sensibilidade}

Para verificar o efeito de algumas variáveis econômicas sobre a distância máxima de transporte foi feita uma simulação. Assim consideraram as principais variáveis: custo de implantação, taxa de juros, preço da terra, produção florestal e preço da madeira, pois estas têm sido as variáveis que mais afetam a rentabilidade dos projetos florestais. Considerando variações entre $-20 \%$ á $+20 \%$ nessas variáveis foi possível identificar quais teriam maior influencia na distância de transporte de madeira. Os indicadores econômicos VPL, TIR e CMP foram as variáveis resultado.

\section{RESULTADOS E DISCUSSÃO}

\subsection{Distância e custo de transporte}

A Figura 1 mostra o custo total de transporte por hectare para os diferentes veículos, considerando-se uma distância de $100 \mathrm{~km}$. Os valores estão variando de US\$1333.33 a US\$1953.49 pelo rodotrem e caminhão truck, respectivamente. Apesar de a capacidade de carga em tonelada do rodotrem e outros veículos maiores ser bem superior à dos caminhões menores como o truck, o que mais limita o transporte da madeira não é o peso e sim o volume. Assim, o volume transportado por esses veículos não é tão discrepante quando a capacidade em tonelada transportada. Por isso, o custo de transporte entre os veículos apresentou pouca variação no geral. Mesmo assim, observou-se que com o caminhão menor seria necessário maior número de viagens por hectare, o que acabaria elevando o seu custo.

A Figura 2 ilustra a distância máxima percorrida pelos diferentes tipos de veículos. Observa-se, nessa figura, que o rodotrem percorre até $226 \mathrm{~km}$ sem que haja prejuízo, distância essa maior que as observadas em outros tipos de veículos. Já o caminhão truck, de menor capacidade de carga que os demais veículos, apresentou a distância máxima viável de $155 \mathrm{~km}$.

\subsection{Análise econômica}

Os indicadores econômicos do projeto florestal para uma distância de transporte de $100 \mathrm{~km}$, utilizando os diferentes tipos de veículos, são apresentados no Quadro 3.

Em todos os tipos de veículos, os indicadores econômicos evidenciam viabilidade do projeto, pois os VPLs apresentaram-se positivos, as TIRs estão acima da taxa de juros e os CMPs estão abaixo do preço da madeira, o que garante a lucratividade do projeto.

Caso não houvesse custo de transporte, e a madeira fosse comercializada ao mesmo preço de US $\$ 24,00 / \mathrm{m}^{3}$, a lucratividade seria bem mais elevada(VPL de US\$1762.10/ ha; TIR de $19,02 \%$ ao ano e CMP de US $\$ 13.21 / \mathrm{m}^{3}$ ). Considerando os diferentes tipos de veículos, os indicadores econômicos são melhores quando o transporte é realizado pelo rodotrem (VPL de US\$984.11/ha; TIR de 14,88\% ao ano e CMP de US $\$ 17.98 / \mathrm{m}^{3}$ ) e piores quando realizados pelo caminhão truck (VPL de US\$622.26/ha; TIR de 12,66\% ao ano e CMP de US $\left.\$ 20.19 / \mathrm{m}^{3}\right)$.

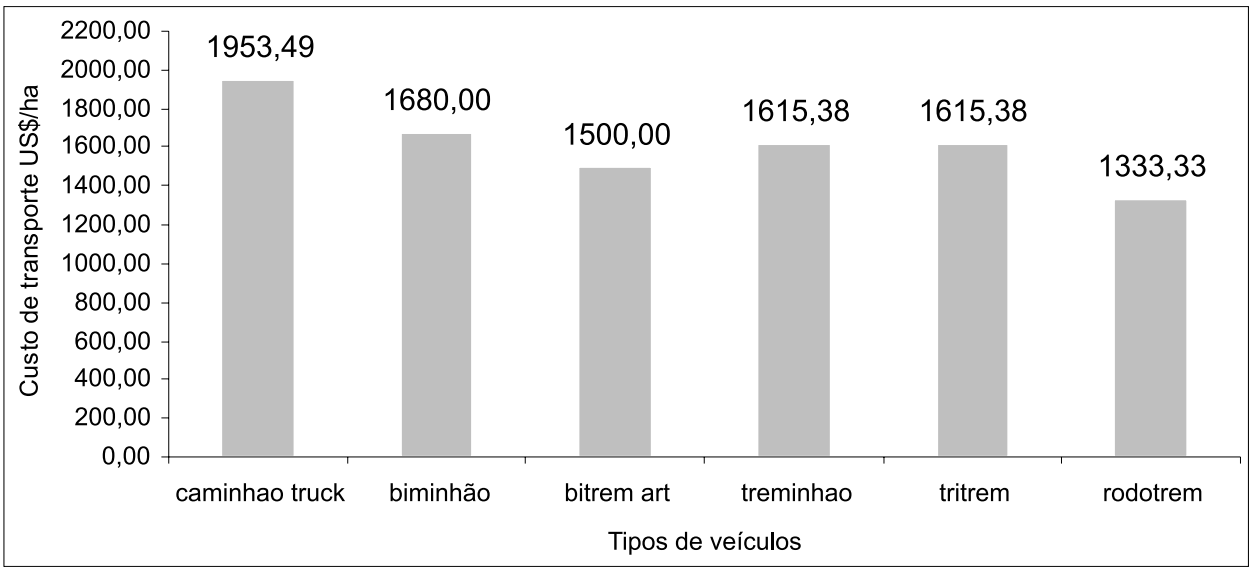

Figura 1 - Custo de transporte (US\$/ha) a uma distância de $100 \mathrm{~km}$ pelos diferentes tipos de veículos. Figure 1-Transport cost (US\$/ha) for a distance of $100 \mathrm{~km}$ for the different types of vehicles.

R. Árvore, Viçosa-MG, v.31, n.6, p.1073-1079, 2007 


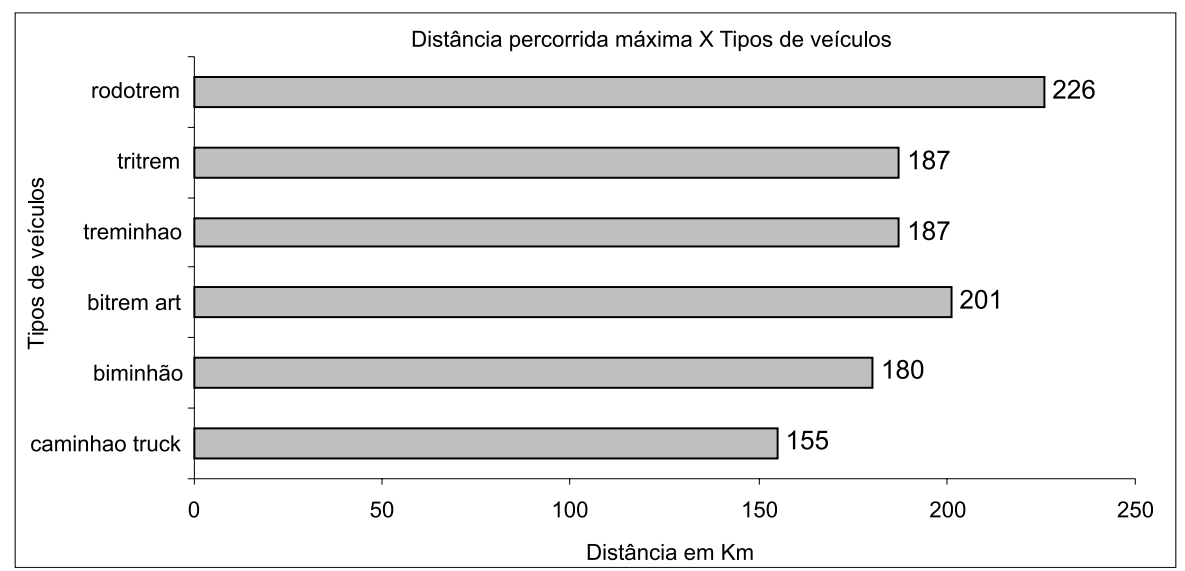

Figura 2 - Distância máxima percorrida pelos diferentes tipos de veículos.

Figure 2-Maximum distance traveled by the different vehicle types.

Quadro 3 - Indicadores econômicos do projeto florestal, considerando-se uma distância de transporte de $100 \mathrm{~km}$, pelos diferentes tipos de veículos

Table 3 - Economic indicators of the forest project considering a distance of $100 \mathrm{~km}$ for the different types of vehicles

\begin{tabular}{lccc}
\hline Tipo de Veículo & $\begin{array}{c}\text { VPL } \\
\text { (US\$ha) }\end{array}$ & $\begin{array}{c}\text { TIR } \\
(\% \text { ao ano })\end{array}$ & $\begin{array}{c}\text { CMP } \\
\left(\text { US } \$ \mathrm{~m}^{3}\right)\end{array}$ \\
\hline Sem custo de transporte* & 1762.10 & 19.02 & 13.21 \\
Caminhao truck & 622.26 & 12.62 & 20.19 \\
Biminhão & 781.83 & 13.65 & 19.21 \\
Bitrem articulado & 886.86 & 14.30 & 18.57 \\
Treminão & 819.54 & 13.89 & 18.98 \\
Tritrem & 819.54 & 13.89 & 18.98 \\
Rodotrem & 984.11 & 14.88 & 17.98 \\
\hline
\end{tabular}

* Considerando o mesmo preço da madeira.

\subsection{Análise de sensibilidade}

A análise de sensibilidade foi feita com relação ao veículo rodotrem e ao caminhão truck, ou seja, o melhor e o pior dentre os diferentes veículos considerados neste estudo.

A análise apontou o efeito do custo de implantação do projeto florestal, da taxa de juros, do preço da terra, da produção florestal e do preço da madeira, na distância de transporte, quando alterados do seu valor-base para valores $20 \%$ menores e $20 \%$ maiores. Nas Figuras 3 e 4, apresentam-se as variações nas distâncias máximas de transporte quando se alteram as demais variáveis do projeto.

Tanto pelo rodotrem quanto pelo caminhão truck, o preço da madeira foi o fator que mais influenciou a distância máxima de transporte. No caso do rodotrem
(Figura 3), aumentando-se o preço da madeira em 20\%, a distância máxima de transporte teve incremento de $44 \%$, aumentando, assim, a sua logística de transporte em relação à situação original. No entanto, se o preço da madeira cair em $20 \%$, a empresa terá que buscar madeira em áreas mais próximas à fábrica para não ter prejuízo.

Também com relação ao caminhão truck (Figura 4), que tem a variável preço da madeira como a mais influente, aumento de $20 \%$ no valor da madeira possibilita à empresa buscar madeira em torno de $44 \%$ mais distante.

A produção de madeira por hectare é a segunda variável que mais afeta a distância máxima de transporte. Se aumentar em $20 \%$ a produção, a empresa pode buscar madeira a uma distância $16 \%$ maior, aproximadamente.

As variáveis preço da terra, taxa de juros e custo de implantação, quando alteradas, afetam a rentabilidade dos reflorestamentos e, portanto, a distância de transporte. Porém, tiveram menor influência que o preço da madeira e a produção.

Se o custo de implantação da floresta aumentar em $20 \%$, a distância de transporte deve ser reduzida em $9 \%$.

Se a taxa de juros aumentar $20 \%$, a distância de transporte deve ser reduzida em aproximadamente $12 \%$.

Se o preço da madeira aumentar em $20 \%$, a distância de transporte deve ser reduzida em aproximadamente $3 \%$, sendo a variável menos influente nessa analise.

R. Árvore, Viçosa-MG, v.31, n.6, p.1073-1079, 2007 


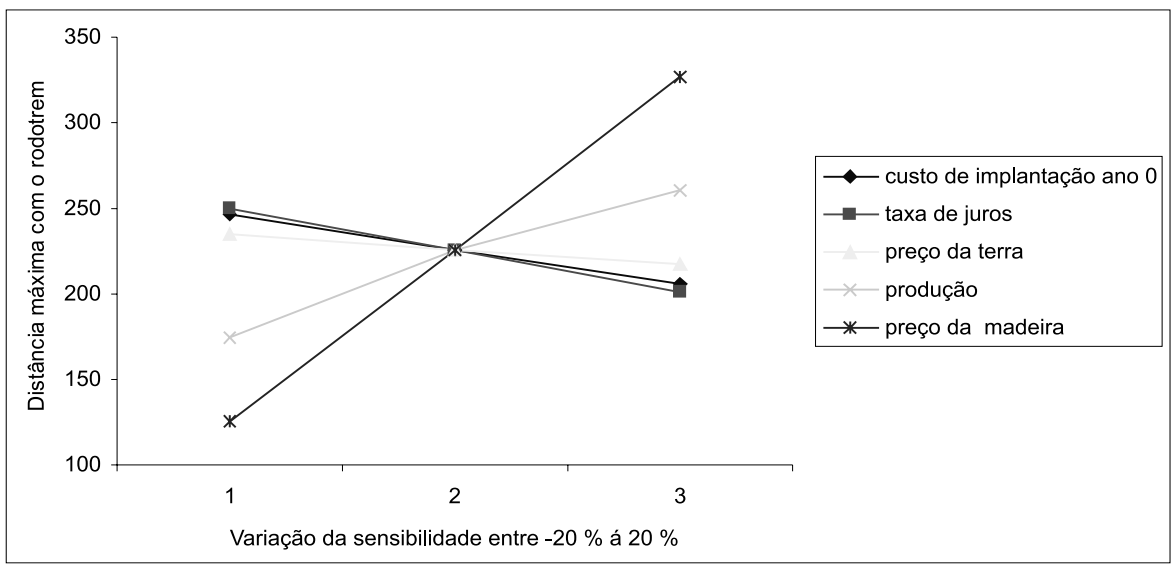

Figura 3 - Influência de alguns fatores na distância máxima de transporte do rodotrem. Figure 3 - Influence of some factors on rodotrem maximum transport distance.

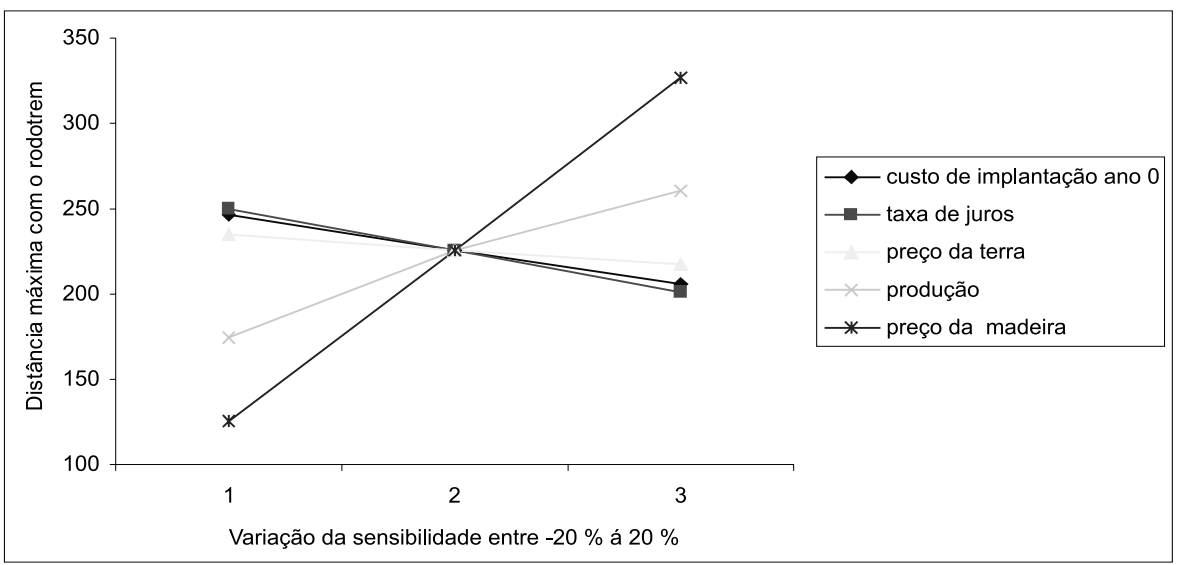

Figura 4 - Influência de alguns fatores na distância máxima de transporte do truck.

Figure 4 - Influence of some factors on small truck transport distance.

\section{CONCLUSÃO}

- Em um raio de transporte de $100 \mathrm{~km}$, é perfeitamente viável implantar reflorestamentos para quaisquer que sejam os veículos de transporte de madeira analisados.

- Dentre os vários tipos de veículos analisados, o rodotrem foi o que apresentou melhor desempenho, pois, dada a sua maior capacidade de carga, seu custo de transporte fica relativamente menor, sendo possível alcançar maior distância de transporte.

- O caminhão truck apresentou o pior desempenho, pois, em uma distância de transporte de $100 \mathrm{~km}$, seu custo ficou $46 \%$ maior que o do rodotrem. E a sua distância viável de transporte é $45,6 \%$ menor que a do rodotrem.

- As variáveis preço da madeira e produção apresentaram maior influência na distância máxima de transporte de madeira.

- As variáveis custo de implantação, taxa de juros e preço da terra, apesar de afetarem a rentabilidade dos projetos florestais, tiveram menor influência na distância máxima de transporte.

- Estudos mais detalhados devem ser conduzidos para identificar quais faixas de distâncias são mais adequadas para cada tipo de veículo, incluindo-se também diferentes categorias de estradas. 


\section{REFERÊNCIAS}

BAESSO, D. P. Estradas rurais:técnicas adequadas de manutenção. Florianópolis: DER, 2003. 236p.

LOPES, E. S. Aplicação do programa SNAP III (scheduling and network analysis program) no planejamento da colheita e do transporte florestal. 2001. 156f. Tese (Doutorado em Ciência Florestal) Universidade Federal de Viçosa, Viçosa, MG, 2001.

LEITE, A. M. P. Análise dos fatores que afetam o desempenho de veículo e o custo de transporte de madeira no distrito florestal do Vale do Rio Doce/ MG. 1992. 105p. Dissertação (Mestrado em Ciência Florestal) - Universidade Federal de Viçosa, Viçosa, MG, 1992.

MACHADO, C. C. Elementos básicos do transporte florestal rodoviário. Viçosa, MG: Editora UFV, 2000. 167p.

NOCE, R. et al. Concentração das exportações no mercado internacional de madeira serrada.

Revista Árvore, v.29, n.3, p.431-437, 2005.
SEIXAS, F. Novas tecnologias no transporte rodoviário de madeira. In: SIMPÓSIO BRASILEIRO SOBRE COLHEITAE TRANSPORTE FLORESTAL, 5., 2001, Porto Seguro.

Anais...Viçosa, MG: Sociedade de Investigação Florestal, 2001. p.1-27.

SILVA, M. L.; FONTES, A. A. Discussão sobre os critérios de avaliação econômica: Valor Presente Líquido (VPL), Valor Anual Equivalente (VAE) e Valor Esperado da Terra (VET). Revista Árvore, v.29, n.6, p.931-936, 2005.

SILVERSIDES, C. R. Survey of truks for raw material transport. World Wood, v.17, n.12, p.42-52, 1976.

SOCIEDADE BRASILEIRADE SILVICULTURA-

SBS. Estatísticas: setor florestal brasileiro; dados sócio-econômicos. Disponível em: (http://www.sbs.org.br/ setor_florestal.htm).Acesso em 16 de out. de 2006.

STEIN, F. R.; RODRIGUES, L. A.; SCHETTINO, S. Sistema de transporte rodoviário da Celulose Nipo Brasileira-CENIBRA. In: SIMPÓSIO BRASILEIRO SOBRECOLHEITAETRANSPORTEFLORESTAL, 5., 2001, Porto Seguro. Anais... Viçosa, MG: Sociedade de Investigação Florestal, 2001. p.109-121. 
